\title{
Towards a Tool for Design Ideation: Insights from Use of SketchStorm
}

\author{
Siân Lindley ${ }^{\alpha}$, Xiang $\mathrm{Cao}^{\beta}$, John Helmes ${ }^{\alpha}$, Richard Morris ${ }^{\varepsilon}$ and Sam Meek ${ }^{\delta}$ \\ aMicrosoft Research Cambridge ${ }^{\beta}$ Microsoft Research Asia ${ }^{E}$ The Open University $\delta$ University of Nottingham \\ Cambridge, UK Beijing, China Milton Keynes, UK Nottingham, UK \\ \{sianl; xiangc;v-johelm\}@microsoft.com; richard.morris@open.ac.uk; psxsm6@nottingham.ac.uk
}

\begin{abstract}
We present findings from a deployment of SketchStorm, a tool for designers that supports sketch in a central canvas, whilst streaming images relating to a search query around the periphery. Our overarching goal was to explore the potential for combining sketching and use of examples, two activities that are associated with design ideation. Initial interviews with designers suggested that a tool that supports encounters with non-designerly content, that supports awareness of what has already been collected, and that allows this content to be laid out, manipulated, and integrated into the process of working out of ideas, would be of value. A month-long deployment allowed us to examine these ideas in more depth, through 'research through prototypes in practice' (Keller et al., 2009). Our findings highlight two ways in which web-based images can be utilised. On the one hand, they can serve as examples and, where this is the case, encounters with them should be rich and memorable, and tools should support a range of actions such as triaging, annotation, and manipulation. On the other hand, images can be used to create a backdrop to on-going activity, so as to underpin serendipitous encounters. Where this is the case, enabling designers to engineer these encounters, so that they are framed by moments of idleness and latent goals, is key.
\end{abstract}

Sketch, image search, image collection, encountering information, flexibility, appropriation.

\section{INTRODUCTION}

It is widely accepted that the early stages of the design process, in which designers generate and explore initial ideas, can have important ramifications on the quality of a final design. This phase of design ideation involves investigating potential problem spaces, considering multiple possibilities, evaluating tentative solutions, and iterating these to allow for the reformulation of solutions (e.g. Bonnardel and Sumner, 1996). $\mathrm{HCl}$ researchers have explored in depth some of the activities associated with design ideation, including sketching, which has been postulated as central to creative output (Buxton, 2007), and the use of examples (Herring et al., 2009), which can serve as a source of inspiration.

This research has typically led to the development of new tools which focus on either sketching or the use of examples. However, recent advances in technology have the potential to draw these two activities closer together. For example, pen-enabled tablet computers offer a means of combining sketch and web search, which is often used when collecting examples (Sharmin et al., 2009). Indeed, innovation may eventually support web searches that are initiated through the act of sketching itself. In this paper, we begin to explore what a technology that combines both processes might offer, and what the challenges of integration are. We follow Keller et al. (2009) in conducting 'research through prototypes in practice' by firstly, developing a tool that allows designers to sketch and collect examples and, secondly, deploying it in the field for a month. In this paper, we report findings regarding the combination of these activities, highlighting implications for tools to support design ideation.

\section{RELATED WORK}

Before detailing our own work, we briefly review research into the process of design, and give an overview of tools that have been developed to support it. Design is generally understood to be an iterative cycle, through which ideas are investigated and successive partial solutions are evaluated (Bonnardel and Sumner, 1996; Schön, 1991). As already noted, examples play an important role in the early stages of this process; their collection is a central part of researching and iterating ideas (Sharmin et al., 2009). The role of sketching has also been highlighted. It has been argued that sketching enhances the restructuring of imagery, which is difficult to perform mentally and is an important element of creativity (Verstijnen et al., 1998). Furthermore, sketches have been highlighted as an external medium that 'talks back' to the designer (Schön, 1991), that supports analogical or metaphorical thought (Goldschmidt, 2003), and that permits sufficient ambiguity for new interpretations to emerge (Purcell and Gero, 1998).

Within $\mathrm{HCl}$, various efforts have been aimed at supporting the generation and representation of ideas, in design work and more broadly. Examples include SILK (Landay and Myers, 1995) and DENIM (Lin et al., 2000), both of which allow designers to 
create interactive user interface prototypes through digital sketching, K-Sketch (Davis et al., 2008), which supports novice animators in creating kinetic animations through simple sketching, and ILoveSketch (Bae et al., 2008), a virtual sketchbook for creating 3D curve models. A second set of projects focuses on providing inspiration, often directly from the web. Examples include InkSeine (Hinckley et al., 2007), which interleaves inking, search and content gathering, Adaptive Ideas (Lee et al., 2010), which supports faceted browsing of example web galleries, and Idea Expander (Wang et al., 2010), which automatically presents pictures to facilitate online brainstorming based on a language processor. Also worth highlighting here is combinFormation (Kerne et al., 2008), a tool that supports information discovery in knowledge creation tasks through the actions of an agent, which collects and composes information for and with the user. Finally, a third set of projects aims to support designers in building and managing collections of content. Freed (Mendels et al., 2011) allows design students to spatially organise their digital collections, while Cabinet (Keller et al., 2009) is an appliance that helps designers merge digital and physical collections of visual material.

These technologies tend to have been evaluated either in constrained settings (Bae et al., 2008; Lee et al., 2010; Lin et al., 2000) or with nonprofessionals, such as students (Lee et al., 2010; Mendels et al., 2011), with the notable exception of Cabinet. Keller et al. (2009) argue for doing "research through prototypes in practice" (p. 85), and deployed their own device with three designers for four weeks. They were particularly struck by the way the device was used to organise existing visual material with the designers' own solutions, highlighting this as an area worthy of further exploration. In this paper, we build upon Keller et al.'s work in two ways. Firstly, we follow their lead in deploying a prototype. This enables us to explore design in practice, and to consider with some degree of rigour the possibilities offered by a technology that supports both sketch and the use of examples. In doing so, we also explore one area that Keller et al. point to as being of especial interest: the possibility for designers to organise existing material with their own solutions, and further, to combine these in a single artefact.

\section{INFORMING THE DESIGN}

To inform our design, we interviewed 12 designers, 7 individually and 5 as a focus group. We focused on their methods for seeking inspiration and developing ideas, their approaches to sketching, and their use of technology, the web and image collections. We interviewed 5 web designers, 3 interaction designers, 1 product designer, 1 graphic designer, and 2 design leads. They were 3 females and 9 males, in the UK and USA, ranging from recent graduates to senior professionals.

Transcripts from the interviews (denoted I) and focus groups (denoted FG) were analysed and key themes identified using open and axial coding (Strauss and Corbin, 1998). Findings that informed our design are presented below.

Firstly, it was clear that designers rarely used the web to directly seek inspiration: "I find it hard to explicitly find inspiration .. at the moment l'm relying on just remembering things that I encounter when I browse" (14); "I don't look for specific explicit inspiration, it's whatever is there .. it's at the whim of whatever someone is serving up on the [RSS] feeds" (FG9). Instead, designers browsed design sites and followed design feeds. These activities had the potential to facilitate encounters with interesting content, which could then be drawn upon later. This resonates with the design studies literature, where it has been posited that new ideas are linked to old situations (including those not related to the current context; Bonnardel, 2000), and that creativity is built upon episodic knowledge (Visser, 1995).

However, search engines, and the web more generally, were noted as being useful when breaking away from familiar sources and those considered "too designerly" (I8). While designers regularly browsed design-related content, they also highlighted this practice as problematic: "creative magazines are a really dangerous trap .. loads of these same ideas emerge, if everybody's working from the same set of references" (I6); "sometimes the ideas are too finished or too strongly themed.. it's completely assembled design" (I8). Similarly, when looking for sources to feed design work, the risk of using content too similar to the intended product was noted. The designers of a web portal avoided looking at similar sites: "it's like pop will eat itself, everything starts to look the same" (FG10). This resonates with Herring et al.'s (2009) observation that examples can result in design fixation, and the web is a way of circumventing this.

A third observation relates to the collections that designers created. As part of their explorations of online content, some designers built image banks that were "scrolled through intermittently", but "going back and browsing all of them" was noted to be a "big problem" (FG9). The content these contained was sometimes explicitly used, for example, 18 drew on his collections for creating mood boards. Lucero (2012) has underlined the value of this activity, and Eckert and Stacey (2000) have shown that collections of content can be used to identify elements or relationships for a new design context, as well as to furnish a vocabulary for thinking about and describing new designs. However, the difficulty of browsing digital collections meant that participants in this study rarely did so if not motivated by a task that would require it. 


\section{SKETCHSTORM}

Drawing on our initial findings and the literature described above, we identified three functions that we wished to explore through a prototype technology. Firstly, as encounters with less designerly content were valued, we chose to support the streaming of images derived from a search engine. Secondly, as designers struggled to revisit their digital collections, but spoke of familiarity with them as being essential to inspiration, we chose to support the streaming of collected materials to reinforce designers' awareness of them. Thirdly, as we aimed to explore the potential for integrating sketch and use of examples, we chose to support the collection, manipulation, laying out and triaging of images, and to incorporate this with sketch functionality.

The subsequent development of our tool, named SketchStorm, was an iterative process that incorporated further interviews with designers in our research group, in order to minimise feature and usability issues prior to deployment. As we wanted to deploy the tool with designers at work, and so learn from its use in practice, it needed to be robust and flexible. Consequently, it was built to run on Microsoft Windows ${ }^{\mathrm{TM}}$, to be used with a Wacom tablet or mouse, and to have different modes that could be appropriated for different purposes: Regular, Thumbnail and Full Screen.

The prototype that we deployed comprised of a canvas for sketching and two image streams on either side, which drew content from the web and from local collections. These drifted past, and could be dragged onto the canvas and used in sketches, or saved. The functionality and interface are described in more detail below.

\subsection{Functionality and interface}

In Regular mode (Fig. 1a), the interface comprises a canvas in the centre and an image stream on each side. The canvas can be used for freehand input while the image streams deliver results from a search query. New images appear at the bottom of the streams and flow upwards at a rate of approximately three seconds per image. This means that images can be encountered somewhat passively, while the user is using the canvas or is engaged in some other activity. A new query can be initiated by typing into the search box or circling a handwritten word on the canvas. In addition, the system can be set to continuously recognise handwriting and use this to update the query. Should the user wish to navigate the streams, they can scroll backwards or forwards, to review prior images or fetch new ones quickly. Double-clicking an image opens a higher-resolution preview.

Both streams can be customised to display content from four sources: Bing (general image search),

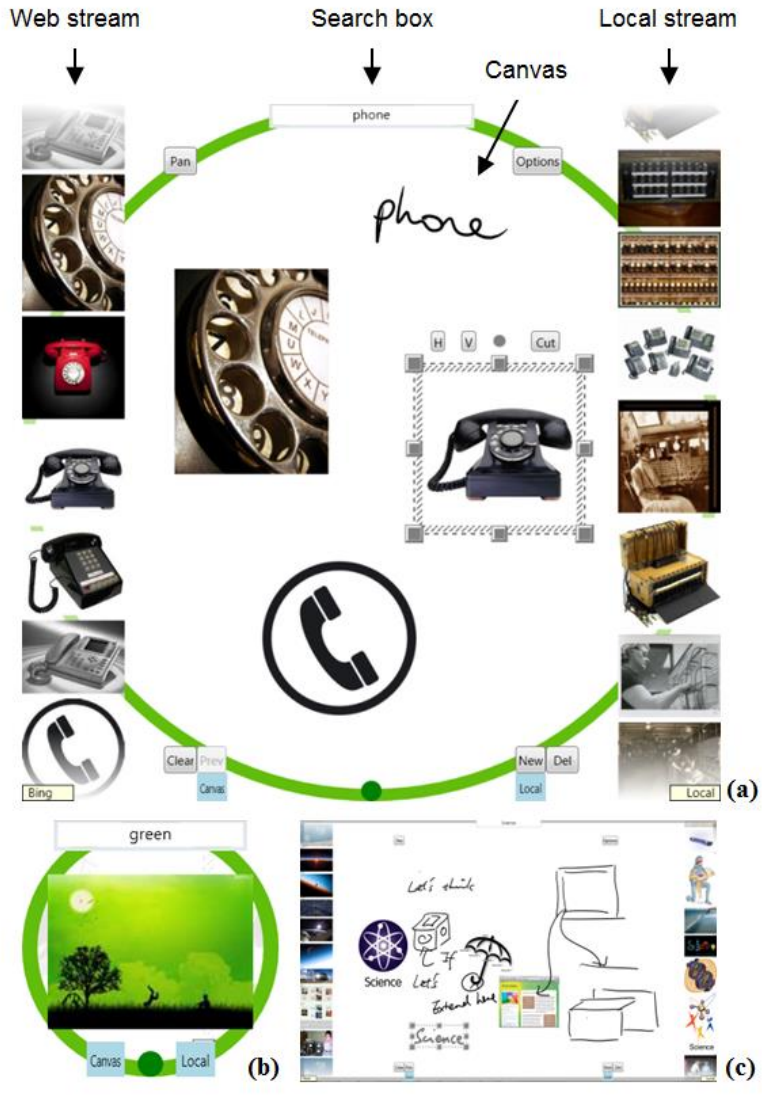

Figure 1: SketchStorm interfaces: (a) Regular (b) Thumbnail (c) Full Screen (images not to scale).

Flickr (user-contributed images), Getty (professional stock images), or a local folder. By default, the left stream delivers results from Bing and the right stream presents images from a local folder. Both streams present content that is relevant to the current search query, but images delivered from a search become gradually less relevant over time, and those from a folder include random images once all those with names or tags that are relevant to the query have been presented. Consequently, the two streams mix directed search and unanticipated browsing, including of one's own image collection. It was hoped that the combination of these two sources could be appropriated to deliver content that is 'less designerly' than design-oriented websites, yet different and less precise than interaction with an image search engine.

Images displayed in the streams can be dragged onto the canvas, where the user can apply basic manipulations such as repositioning, resizing, rotating, horizontal/vertical flips, and cutting out sections. In addition, users can draw on top of images, allowing them to combine sketches and image elements when exploring ideas. Elements on the canvas, either alone or in combination, can be saved to the local folder, as can images in the web stream, which can be dragged there directly. Saved content is tagged with the original image file name and the search query used to retrieve it; sketches are tagged with any handwritten notes. The addition 
of these tags supports later re-finding and presentation in the local stream; if the same search query is entered twice, images saved during the first search will be presented in the local stream during the second search.

We anticipated that different designers would have different needs for a tool like SketchStorm. Two additional modes were developed to place more or less emphasis on sketching with and around images. When SketchStorm is resized below a certain threshold, it switches to Thumbnail mode (Fig. 1b), where both the canvas and the image streams are hidden, and only one image is displayed at a time. This image can be set to come from either one of the streams or both. Thumbnail mode allows SketchStorm to be used in a peripheral fashion, but continues to support encounters with and saving of images; images can still be placed on the canvas or in the local folder via two drop boxes. Additionally, to support placement in the periphery of the screen, SketchStorm sits 'on top' of other applications while in Thumbnail and Regular mode. The final mode is Full Screen, accessed by double-clicking the light green border (Fig. 1c). Here, the maximum canvas area and number of streamed images is provided.

In both Regular and Full Screen mode, the user can pan and zoom the canvas and flip between different pages using the Prev and Next buttons. Individual sketches or the entire canvas can be deleted using the Del and Clear buttons.

\section{FIELD DEPLOYMENT}

As already mentioned, little of the prior work in $\mathrm{HCl}$ aimed at supporting design ideation has resulted in technologies deployed with designers at work. This was an important goal with SketchStorm; we wanted to learn from how it would be used in design practice. Accordingly, we deployed the prototype with nine designers to use on their work computers over the course of a month. In this section, we focus on how SketchStorm was (and was not) incorporated into processes associated with design ideation, and what this can tell us about designing new tools aimed at supporting this.

\subsection{Participants}

Our participants were six males and three females, ranging from recent graduates to senior design leads. There were three in China, three in the UK, two in the USA and one in the Netherlands. Four (D4, D5, D7, D8) were interviewed as part of our initial study (14, I5, FG7, I8). D1-3 were working on concept development, D4 prototypes user interfaces, D5 is a systems designer, D6 is a touch interaction designer, D7 designs wireframes and modules for the web, D8 is a graphic designer for print and web, and D9 is a design researcher.

\subsection{Method}

The designers were interviewed using semistructured interviews on three occasions: at the beginning of the deployment, for us to demonstrate the prototype and find out about their existing design practice; after one week, to understand initial impressions of SketchStorm and how it fitted their design practice; and after one month, to understand if and how use had changed over time. Questions included what circumstances triggered use of the prototype, whether it had become part of a routine, whether it had disrupted the designer's practice, whether it was used to discover visual content, whether it was used to store or archive material, whether it was used for sketching, and how it was used across the three modes of the interface. Interviews typically lasted between 30 minutes and one hour and were conducted either in person or via internet calls, in the latter case facilitated by screen sharing. Canvasses and images saved by designers were used to ground the discussion.

\subsection{Analysis}

The interviews were transcribed and analysed in order to identify emergent themes using open and axial coding (Strauss and Corbin, 1998). It is worth highlighting that while some of the designers used SketchStorm enthusiastically (and continued to do so after the period of study), others did not so readily take to it. Our analysis focuses as much on reasons underpinning adoption of SketchStorm as on reasons for low usage. As the analysis developed, the core theme of 'mastery of a tool', which was relevant to both types of response, was identified and iterated on. We now describe four key sets of findings that emerged from this analysis.

\subsection{Findings}

\subsubsection{Inspiration 'seeking' can be secondary}

The first theme we report relates to how designers appropriated SketchStorm to encounter interesting images. As noted earlier, inspiration is rarely explicitly 'sought'. Nevertheless, designers were able to use SketchStorm to encounter interesting content serendipitously, as well as to build collections of images that would serve as a resource in the design process.

If we consider use of SketchStorm to underpin serendipitous encounters first, it is interesting to see how this circumstance was engineered by designers. Indeed, this highlights a subtle contrast with our initial interviews, which indicated that designers felt unable to 'search' for inspiration. In contrast, our deployment showed that designers could, and did, explicitly facilitate encounters with interesting content. This was achieved by positioning SketchStorm at the periphery of the screen and using search queries such as 'sunshine', 
'sky' and 'green' to stream content. Furthermore, D4 developed a tactic to display random images; by selecting an arbitrary part of a sketch to be (mis)interpreted by the handwriting recognition, she triggered uncalculated searches. While such behaviours were described in quite passive terms, such as "procrastination" (D2), "idleness" (D4), and "like looking outside of the window" (D1), the experience was, in a sense, framed quite deliberately, and was sometimes driven by a specific aim. For example, D1 set SketchStorm to run "inspirationally in the background" while sketching on paper ("you keep an eye on what's happening on the screen ... it's like you catch what's interesting"), and D9 placed it on the margins of his monitor to prompt some work that needed to be done whilst engaging in a separate task: "It was the pressure to keep thinking about something ... I had the idea at the beginning of the day that I wanted to start thinking about logos. So at the beginning I put the widget on there with the keyword 'phone switchboard' .. this one popped up and I started to sketch" (see Fig. 2).
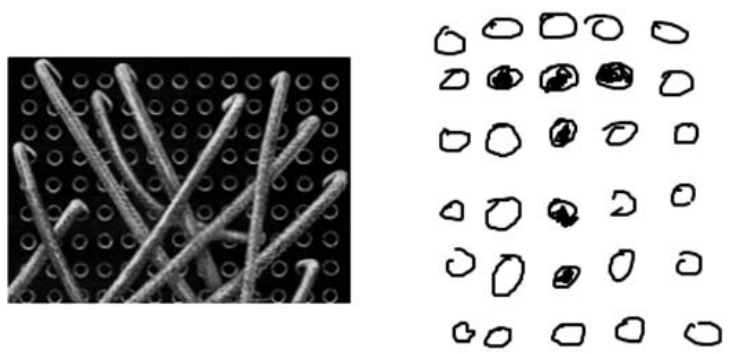

Figure 2: Image of a switchboard and sketch of a logo.

Despite this use of SketchStorm to stream content, few of our participants used it to then build collections. The exception here was D8, who undertook directed research for each project he worked on. He compared the passive search experience offered by SketchStorm to "cruise control" in a car, and much preferred it to the "tedious" experience of "trawling an endless amount of images" via a search engine. He saw SketchStorm as offering an "opportunity to step back" when momentarily stuck on some other task, allowing him to "multi-task" and find more images with reduced effort: "the search that I did before .. turned out 80 images, with SketchStorm I got 184, it's a massive difference".

Common to these different examples is that, by repositioning image search as a peripheral activity, designers could use it to encounter inspirational content in a way that is difficult with search tools. 'Searching' for inspiration was accomplished as a secondary, yet intentional, activity.

5.4.2. The design process is demarcated by tools The second theme of our analysis relates to how SketchStorm performed and was understood as a tool that slotted into the design process, a finding that is particularly relevant to our aim of considering the possibilities offered by integrating sketch and use of examples. Indeed, and counter to the notion of integration, our findings highlighted that designers value having the "right kind of tool for the right kind of job" (D7), one that is specifically tailored to a given activity. One consequence of the combination of sketch and image search in SketchStorm was that it was seen as "too broad" (D2). As D6 noted, "I haven't gotten into a rhythm where .. it was the perfect tool for a specific task".

D6's observation is partly bound up with the technical limitations of our prototype; digital sketching is not as satisfying as sketching on paper (described as "familiar" (D3), having a "rhythm"(D6), even as making a "nice noise" (D1)), and SketchStorm was not as powerful as other, existing, digital sketch technologies (such as Illustrator, noted by D2 for providing "this power" that allowed him to "play", "reformulate" and "transform" his work in a manner that was "comfortable", "easy" and "fast"). Furthermore, while we had not intended to create a perfect search tool with SketchStorm, its lack of exactness meant that it sometimes drew unfavourable comparisons with other tools used by the designers, which were underpinned by values such as "precision" and "control" (D2). Nevertheless, the deployment of SketchStorm allowed us to consider whether combining sketching and use of examples could be of value more broadly. Indeed, their integration was a factor that divided opinion throughout the study, as we will now illustrate.

Where integration was viewed positively, reduced effort was noted as one of the most straightforward benefits that SketchStorm offered. It made it easy to "integrate picture material into sketches" (D4). D9 described how he used images sourced from SketchStorm as the backdrop for sketches of a watering system (Fig. 3), saying "I wouldn't have taken the effort to start Photoshop and search an image .. and save it and open it .. and create a layer to start sketching on it", and D4 integrated web images into the design of an interface (Fig. 4). She noted that it was valuable to be able to save these image and sketch combinations, as well as the component parts themselves: "you can use the whole thing that you draw, so the integration of that one [image] with the actual sketch can be then used as a whole .. which I can't do on paper" (D4).

More fundamentally, D8 felt that SketchStorm offered a way for him to combine elements of the design process that were related but normally done separately: "those three things, you know, the collecting of images, the reviewing of images and making notes are all done at the same time and at the same place for me, and exactly the same, definitely the same stage of my project". As already noted, he used the tool for searching for images for 
specific projects, a highly structured and timeconsuming process. His normal practice was to search for images using a search engine, save these to project-specific reference folders, and then use them in the process of building ideas and clarifying communication with clients. SketchStorm offered advantages over his usual way of doing this. On noticing an interesting image, he would move it to the local folder, or if it was particularly significant, onto the canvas as well. From there he could outline "parts of the image that I like by drawing over the top", and make "notes, and .. a little montage of different images and noted things that I like about that image" (Fig. 5a). SketchStorm offered a "good way to put all the found images together in one place .. and view them all at the same time" (Fig. 5b), resulting in a "board of visual thinking .. loads of visual reference material based on key words which I then use to create designs".
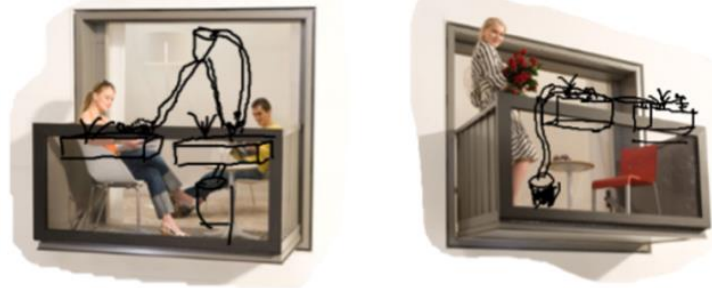

Figure 3: Combinations of image and drawing used as a backdrop for sketches of a balcony watering system.
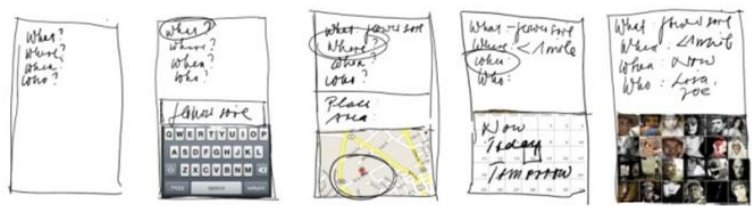

Figure 4: Sketches of interfaces utilising web images.
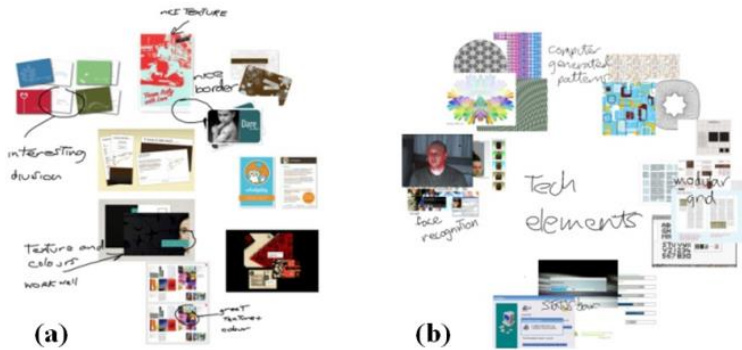

Figure 5: Images (a) annotated to highlight points of interest and (b) arranged as an "idea board".

While for D8 SketchStorm was "definitely a workrelated tool" because it allowed him to combine activities he normally had to do separately, other designers saw SketchStorm as mixing activities that were better kept separate. As D6 explains, "I'll do some sketches or l'll do some thinking and then .. my second step .. is like finding the resources, finding the images, and then the last step is kind of .. execution, finalising things .. it tries to merge all those into one place". Relatedly, designers showed little interest in creating sketches that could be exported to the next phase of the design process. Digitising a sketch was part of the process of refining ideas, and if the opportunity to create high-fidelity sketches too early on was present, this could be seen as introducing the pressure to create something of too high quality: "I would have to spend more time on sketches and then it defeats the purpose" (D4). The demarcation of the design process was bound up with the use of separate tools at different stages.

\subsubsection{There is little value in revisiting collections}

A third theme, which was common across all participants, was that little value was perceived in the ability to revisit already-collected materials. Most changed the settings for the image stream that, by default, displayed local content, to show images derived from the web. This reflected more general practices surrounding the perusal of archives, which was also noted as being rare.

One of the reasons underpinning this was quite straightforward. Some designers simply did not have an archive that could be used to feed SketchStorm. Collections might be on "scattered hard drives and various computers" (D7), or had been abandoned because they were difficult to maintain and search: "the folder just got too big, I wasn't really referring back to it .. if you just keep a general folder everything can get out of date so quickly, it's just low value" (D8). The web offered a valuable alternative here: "deviantART and Dropular have such a huge library stored online that I can enter a search term and know that they are going to find it" (D7). This resonates with prior work (Sharmin et al., 2009), where it has been noted that the cost of access via the web is lower than retrieval from personal collections.

However, even designers who did archive tended not to "browse through" (D1) their collections. Instead, they would look to them only if they remembered content worth retrieving: "if I could recall that I found something .. I would go back, but I wouldn't do it to get inspiration and start browsing those folders" (D9). In general, the web was a first port of call, with archives being "a safe point" (D2), like "my collection of tools in my garage ... I don't need it all that often, but when I do .. it's awesome to have" (D5).

An interesting observation that came out of these discussions is that the act of saving images, within SketchStorm and more generally, was felt to make them memorable in itself: "sometimes it's just the effect of going through the process of saving it made it more memorable, so sometimes I don't need to go back"(D4). This has practical implications if archived material is recalled rather than revisited: "you're thinking about things you've seen somewhere, or 
you remember what [is ..] relevant" (D4). Support for recall of material, rather than re-finding it, may be paramount in design.

\subsubsection{Image search is not just about imagery}

The final theme relates to the content that SketchStorm dealt with: images. SketchStorm was built around a visual search mechanism that delivers images as streams that are linked by a search query but are otherwise unrelated to one another. We intended that this would support interesting encounters with images and wondered if surprising juxtapositions might be of use when building ideas, in the same way that brainstorming techniques utilise synonyms and place material out of context. However, our data demonstrate that the context in which images are encountered is important. Context provides narrative and a sense of currency, qualities that SketchStorm were said to remove. For the designers that we interviewed, images were typically encountered on blogs, articles, and in design forums. Delivering images outside of these sources was described as "like news without any context" (D6). Furthermore, presenting images in isolation meant that knowledge of the source was lost. Source underpinned a sense of "freshness" (D2) and trust: "the ones on the blog .. are picked by human beings with similar design interests, when you search for a term, you get different results than if you ask someone what is inspiring to them; they'll come up with things that you would have never thought to search" (D6). Thus, contemporary and curated sources gave a sense of being up to date.

These findings may at first glance seem odd; designers clearly encounter images out of context whenever they use image search engines (which they did, frequently). However, the designers that we studied do not generally use search tools as a way of discovering or encountering interesting material; this is something that was achieved through browsing. Instead, search tools were used to specifically find an image for a particular reason. Two factors are key here. Firstly, the usual process of image search was noted for giving a sense that one is exploring and understanding a space through the ability to dynamically alter and refine search terms. Images in search engines were viewed like signposts, allowing one to navigate and make sense of an online landscape: "when you do searches through Google Images or Flickr or whatever you constantly adapt your keywords to find more specific images" (D9). Secondly, designers wish to know much more about an image than simply what it looks like when searching, and these requirements are bound up with what they intend to do with that image. Images are materials that can be printed, projected, or shared with others, and when this is the case properties such as image resolution or the provision of a creative commons license are central: "if I have to make a presentation it should be at least
.. 800 by 600 [pixels], if I have to print it, it should be even bigger, so for .. the way I use images .. this is key" (D1).

Of course, SketchStorm does give some indication of context via the sources that images are streamed from. Indeed, the fact that Flickr was shaped by "people passionate about photography" (D1) meant that it was favoured by some: "someone's selected images to put them on there" (D4). However, the way in which the images were displayed was felt to undermine a sense of narrative. Interestingly, this was even true of the local stream. D2 reflected on his own local folder, containing images from different designers: "each of them has one very specific type of work, and I like to visualise them separately because it doesn't make sense for me to combine them". He went on to describe their combination in SketchStorm as "like mixing horror movies with comedy .. it's strange to see them together". This contrasts with images viewed within folders, where the materials can be made sense of as a collection.

\section{DISCUSSION}

In this section we consider what our deployment of SketchStorm reveals for the development of tools for design ideation, as well as for their deployment in design settings.

\subsection{Implications for design}

Our first aim in designing SketchStorm was to support encounters with interesting and less designerly images, in line with the notion that inspiration is not explicitly sought. However, the deployment revealed that, by repositioning search as secondary, designers were able to engage in quite deliberate acts of seeking inspirational content. Furthermore, this was often done to underpin encounters in moments of inactivity. This resonates with prior work on creativity, which suggests that insights often occur when breaking the workflow (Keller et al., 2009) or when one is idle, and that these insights are preceded by periods of preparation and incubation (Csikszentmihalyi and Sawyer, 1995). Similarly, attempts to design for serendipity highlight the importance of identifying information that is relevant to a latent goal (de Bruijn and Spence, 2008). This has led André et al. (2009) to highlight the notion of '(un)serendipity'; they argue that serendipitous discoveries are the product of mental preparation and of an open and questioning mind. Our findings lend support to this view, and highlight the value of supporting designers in engineering situations that allow them to make the most of idle moments, and to peripherally attend to latent goals. Through SketchStorm, they could exert control over when to stream content, in line with when they expected to be able to respond to it in an open and insightful way. 
However, while SketchStorm could be used to potentially engineer interesting encounters with images, this was somewhat undermined by the lack of context it provided in relation to these. By removing details such as image source, SketchStorm was unable to provide the sense of being 'connected' to the world that designers associated with browsing the web. This suggests the importance of semantics, and the notion of journeying, when images form a backdrop to other activities. Semantics are not typically associated with image search, with tools such as Google Images allowing the user to filter by aesthetic qualities such as size, colour and 'type' (face, photo, clip art or line drawing). In contrast, design for serendipitous encounters has highlighted the possibility of incorporating semantic search (Maxwell et al., 2012), and of making interesting connections (André et al., 2009). Our findings suggest that semantic search tools could also be used in the generation of image streams, by creating threads of images that are meaningfully connected and that allow ideas to be explored.

The use of SketchStorm to support serendipitous encounters contrasts with the way in which it was used for more focused acts of collecting examples and researching a problem space. Here, images might be thought of as artefacts to be gathered and manipulated, combined and annotated. Ways of supporting these activities have been highlighted before; our findings are in line with analyses of the ways in which existing tools, such as mood boards, are used (Lucero, 2012), as well with recommendations that highlight the need to support the management and laying out of collected examples (Keller et al., 2009; Mendels et al., 2011) and their flexible annotation (Herring et al., 2009; Sharmin et al., 2009). We will not reiterate those arguments here. However, we do think it worth highlighting that, while most research has positioned annotation as being of value at the moment of retrieval, the designers in our study showed little interest in revisiting their own collections. Instead, and as Sharmin et al. also report, inspiration was often seeded from memory.

Therefore, we argue for the importance of making encounters with content memorable, by supporting richer ways of interacting with it at the moment of collection. In this view, actions such as annotation and tagging might be valued because they offer a deeper means of engaging with an image, rather than because they may later support re-finding. This proposal draws on findings from psychology, which show that deeper cognitive processing of stimuli supports better recall of it in the future (Craik and Lockhart, 1972), as well as research on collecting, which suggests that the act of collecting in itself is a way of creating meaning (Koh and Kerne, 2006). Other possibilities for deeper engagement include revealing more information about the images themselves, such as their online context or source, which may serve the additional purpose of avoiding problems associated with the lack of context provided by SketchStorm. Collectively, these features may enable the creation of rich collections that are memorable to their curators and that can be perused by others, the importance of the latter being highlighted by Sharmin et al. (2009). It is also worth noting that more complex features would fit the distinctions that designers made with regard to images when interacting with them through SketchStorm, for example by pulling them onto the canvas or into a folder, depending on what they intended to do with them. The challenge here is to support the deeper processing that could allow designers to imbue their materials with meaning, whilst keeping the interface simple and intuitive.

As a final observation, the distinction between images as artefacts to be used vs. as underpinning serendipitous encounters also relates to our aim to explore the potential for integrating sketch and use of examples. Here we saw instances demonstrating the usefulness of placing sketch in the digital realm for working out ideas and design solutions, but the word 'sketch' does not totally capture the different activities that our participants engaged in. While on the one hand, designers used the sketch functionality to make notes or annotate mood boards, on the other, they integrated images into sketches themselves, or sketched on paper whilst SketchStorm streamed images in their peripheral vision. Both processes are ways of exploring ideas (see Lucero, 2012, for a consideration of how sketching is similar to creating mood boards) and designers in our study were able to appropriate SketchStorm for both purposes. Yet it may be of value to attend to them separately when developing tools for design ideation.

This is because, as our study makes clear, designers use different tools as a way of demarcating stages of the design process: the adoption of specialised tools, from sketches on paper to digital mock-ups, functioning prototypes and eventual deliverables, was a progression that reflected the narrowing of the problem space and the refining of ideas. A tool for sketch might focus on providing a means of flexibly and powerfully working through ideas, for example by offering greater support for the malleability of digital materials through colour manipulations, or by supporting richer editing and mashing up of images copied onto the canvas. In contrast, a tool for dealing with examples might offer ways of visualising collections and providing notes that are specific to points within an image.

This runs counter to another assumption that underpinned our design. We intentionally developed SketchStorm as a flexible tool, with the expectation that this would fit the openness that characterises 
the early design process, and further, support appropriation in a profession that encompasses a range of specialisms and methods. What we observed, however, was that designers wanted a tool to suit a particular purpose and that was tailored accordingly. The designers that used SketchStorm the most saw it as a work-related tool that opened up new possibilities within their existing practice. Those that saw SketchStorm as being 'too broad' felt that it failed to offer the power and mastery that other tools, both software and pen and paper, enabled.

\subsection{Implications for research}

Related to the above, in our deployment we adopted a strategy that no doubt compounded the perception of SketchStorm as being too open: we were unwilling to specify what it was for. In reporting on their own research through prototypes in practice, Keller et al. (2009) draw on the idea that a design tool reveals itself only in use. They argue that the open-ended structure of the deployment of Cabinet led to many different uses and interpretations of the device. In this study, we also found that designers put SketchStorm to a variety of uses. Furthermore, those uses that we had expected were often underrepresented, whereas others emerged that had not been anticipated.

However, in some cases, our experience resonated more with that reported by Gaver et al. (2009). They note that, although intended to encourage appropriation, a lack of specificity can sometimes backfire. We speculate that openness was particularly problematic in this study because of the workplace setting. Participants were trying to find a role for SketchStorm in an existent toolkit of sophisticated software and pen and paper, an established workflow, and a set of expectations regarding how image search and digital sketch should work. Unlike many technologies that are deliberately ambiguous (e.g. Gaver et al., 2003), it was essential that SketchStorm should slot into current practice and provide some kind of advantage over available tools. So while some designers noted improvements in efficiency and the ease with which they could combine image and sketch, the lack of a transparent advantage over existing practices meant that for others, use of SketchStorm was problematic.

The challenges of testing technologies with designers at work are perhaps made most evident through the lack of reported deployments in the literature; Cabinet is the exception to the rule here. While our own deployment was certainly valuable, with SketchStorm serving as a resource for discussion about design practice, future deployments of tools for design ideation may be more enthusiastically received if they are more precisely focused, catering for a particular design specialism or activity. One possibility here would be to prototype a range of plug-ins for existing tools, in order to flexibly introduce specific features, while removing the need for designers to adopt a totally new technology. Alternatively, taking an approach akin to that outlined by Hutchinson et al. (2003), where technology probes are deployed with a view to being iterated upon and improved with participant input, could be of value when working in this space.

\section{CONCLUSION}

We have presented insights from a field deployment of SketchStorm, a tool to support design ideation that combines sketch and use of examples. Our findings suggest that while web-based tools can and do support encounters with interesting image-based content, this content should be treated in two distinct ways. On the one hand, images can be used as examples to be kept or drawn upon in the future. Where this is the case, encounters with images should be made rich and memorable, and tools should support a range of actions such as triaging, annotation, and manipulation. On the other hand, images can be used to create a backdrop to ongoing activity, so as to potentially enable serendipitous encounters. Here it is important to allow designers to engineer the nature of those encounters, so that they can underpin moments of idleness and be framed with regard to latent goals. Our findings also emphasise that tools for design should reflect the demarcation of the design process, supporting a progression from early ideas to an eventual solution. Thus, simply integrating functionality for sketch and the use of examples can prove problematic, but combining specific aspects of these activities, such as sketching to a stream of inspirational content, or annotating images to be used as examples, could serve to reinforce the two ways of treating web-based images we highlight here, opening up rich possibilities for design.

\section{REFERENCES}

André, P., schraefel, m.c., Teevan, J., Dumais, S. Discovery is never by chance: Designing for (un)serendipity. (2009) C\&C, Berkeley, CA, USA, 305-314. ACM, NY.

Bae, S.-H., Balakrishnan, R., Singh, K. (2008) ILoveSketch: As-natural-as-possible sketching system for creating 3D curve models. UIST, Monterey, CA, USA, 2008, 151-160. ACM, NY.

Bonnardel, N. (2000) Towards understanding and supporting creativity in design: Analogies in a constrained cognitive environment. KnowledgeBased Systems, 13, 505-513.

Bonnardel, N., Sumner, T. (1996) Supporting evaluation in design. Acta Psychologica, 91, 221244. 
Buxton, B. (2007) Sketching User Experiences. Morgan Kaufmann, London.

Craik, F.I.M., Lockhart, R.S. (1972) Levels of processing: A framework for memory research. Journal of Verbal Learning and Verbal Behavior, 11, 671-684.

Csikszentmihalyi, M., Sawyer, K. (1995) Creative insight: The social dimension of a solitary moment. In R.J. Sternberg, J.E. Davidson (eds), The Nature of Insight. MIT Press, Cambridge, MA.

Davis, R.C., Colwell, B., Landay, J.A. (2008) KSketch: A 'kinetic' sketch pad for novice animators. CHI, Florence, Italy, 2008, 413-422. ACM, NY.

de Bruijn, O., Spence, R. (2008) A new framework for theory-based interaction design applied to serendipitous information retrieval. ACM Transactions on Information Systems, 15, 1, 5.

Eckert, C., Stacey, M. (2000) Sources of inspiration: A language of design. Design Studies, 21, 523538.

Gaver, W.W., Beaver, J., Benford, S. (2003) Ambiguity as a resource for design. CHI, Ft. Lauderdale, Florida, USA, 233-240. ACM, NY.

Gaver, W.W., Bowers, J., Kerridge, T., Boucher, A., Jarvis, N. (2009) Anatomy of a failure: How we knew when our design went wrong, and what we learned from it. CHI, Boston, MA, USA, 22132222. ACM, NY.

Goldschmidt, G. (2003) The backtalk of selfgenerated sketches. Design Issues, 19, 72-88.

Herring, S.R., Chang, C.-C., Krantzler, J., Bailey, B.P. (2009) Getting inspired! Understanding how and why examples are used in creative design practice. CHI, Boston, MA, USA, 87-96. ACM, NY.

Hinckley, K., Zhao, S., Sarin, R., Baudisch, P., Cutrell, E., Shilman, M., Tan, D. (2007) InkSeine: In situ search for active note taking. CHI, San Jose, California, USA, 251-260. ACM, NY.

Hutchinson, $\mathrm{H}$. et al. (2003) Technology probes: inspiring design for and with families. $\mathrm{CHI}$, Ft. Lauderdale, Florida, USA, 17-24. ACM, NY.

Keller, I., Visser, F.S., van der Lugt, R., Stappers, P.J. (2009) Collecting with Cabinet: Or how designers organise visual material, researched through an experiential prototype. Design Studies, 30, 1, 69-86.

Kerne, A., Koh, E., Smith, S.M., Webb, A., Dworaczyk, B. (2008) combinFormation: Mixedinitiative composition of image and text surrogates promotes information discovery. ACM Transactions on Information Systems, 27, 1-45.
Koh, E., Kerne, A. (2006) I keep collecting: College students build and utilize collections in spite of breakdowns. ECDL, Alicante, Spain, 303-314. Springer-Verlag, Berlin Heidelberg.

Landay, J.A., Myers, B.A. (1995) Interactive sketching for the early stages of user interface design. CHI, Denver, Colorado, USA, 43-50. ACM, NY.

Lee, B., Srivastava, S., Kumar, R., Brafman, R., Klemmer, S.R. (2010) Designing with interactive example galleries. CHI, Atlanta, Georgia, USA, 2257-2266. ACM, NY.

Lin, J., Newman, M.W., Hong, J.I., Landay, J.A. (2000) DENIM: Finding a tighter fit between tools and practice for web site design. CHI, The Hague, The Netherlands, 510-517. ACM, NY.

Lucero, A. (2012) Framing, aligning, paradoxing, abstracting, and directing: How design mood boards work. DIS, Newcastle Upon Tyne, UK, 438-447. ACM, NY.

Maxwell, D., Woods, M., Makri, S., Bental, D., Kefalidou, G., Sharples, S. (2012) Designing a semantic sketchbook to create opportunities for serendipity. BCS HCl, Birmingham, UK, 2012, 357-362. British Computer Society.

Mendels, P., Frens, J., Overbeeke, K. (2011) Freed: A system for creating multiple views of a digital collection during the design process. $\mathrm{CHI}$, Vancouver, BC, Canada, 2011, 1481-1490. ACM, NY.

Purcell, A.T., Gero, J.S. (1998) Drawings and the design process. Design Studies, 19, 389-430.

Schön, D.A. (1991) The Reflective Practitioner. Ashgate, London.

Sharmin, M., Bailey, B.P., Coats, C., Hamilton, K. (2009) Understanding knowledge management practices for early design activity and its implications for reuse. CHI, Boston, MA, USA, 2367-2376. ACM, NY.

Strauss, A.C., Corbin, J. (1998) Basics of Qualitative Research. Sage, London.

Verstijnen, I.M., Hennessey, J.M., van Leeuwen, C., Hamel, R., Goldschmidt, G. (1998) Sketching and creative discovery. Design Studies, 19, 519-546.

Visser, W. (1995) Use of episodic knowledge and information in design problem solving. Design Studies, 16, 171-187.

Wang, H.-C., Cosley, D., Fussell, S.R. (2010) Idea Expander: Supporting group brainstorming with conversationally triggered visual thinking stimuli. CSCW, Savannah, Georgia, USA, 103-106. ACM, NY. 